Tecno Lógicas

ISSN 0123-7799

Vol. 17, No. 32, pp. 97-109

Enero-junio de 2014
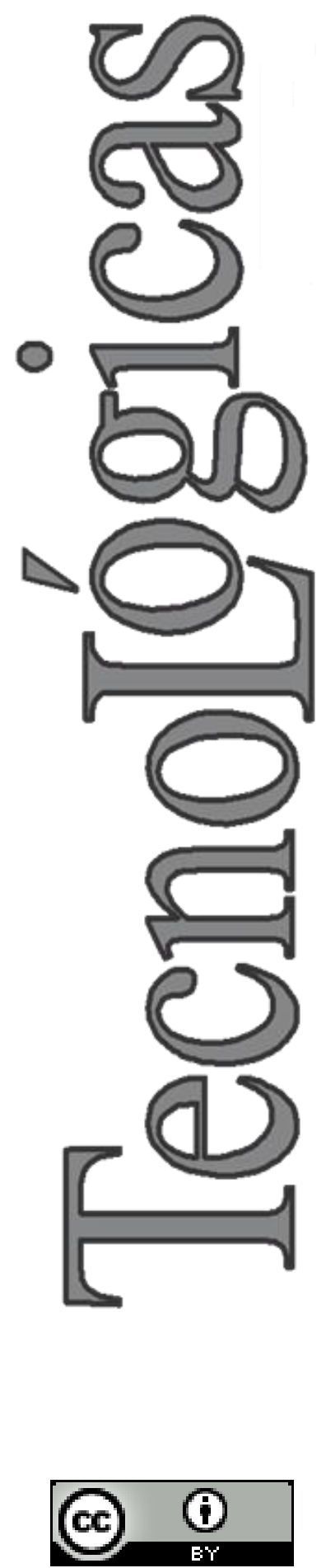

\section{Diseño y construcción de una estación de clasificación automática con visión de máquina}

\section{Design and construction of automatic sorting station with machine vision}

\author{
Oscar D. Velasco-Delgado ${ }^{1}$, María F. Pérez-Sandoval², \\ Juan F. Flórez- Marulanda ${ }^{3}$
}

Recibido: 11 de octubre de 2013, Aceptado: 12 de diciembre de 2013

Como citar / How to cite

O. D. Velasco-Delgado, M. F. Pérez-Sandoval y J. F. Flórez- Marulanda, "Diseño y construcción de una estación de clasificación automática con visión de máquina”, Tecno Lógicas, vol. 17, no. 32, pp. 97-109, 2014.

1 Ingeniero en Automática Industrial, Departamento de Electrónica, Instrumentación y Control, Facultad de Ingeniería en Electrónica y Telecomunicaciones, Universidad del Cauca, Popayán-Colombia, ovelasco@unicauca.edu.co

2 Ingeniera en Automática Industrial, Departamento de Electrónica, Instrumentación y Control, Facultad de Ingeniería en Electrónica y Telecomunicaciones, Universidad del Cauca, Popayán-Colombia, mafep@unicauca.edu.co

3 Magister en Electrónica y Telecomunicaciones, Departamento de Electrónica, Instrumentación y Control, Facultad de Ingeniería en Electrónica y Telecomunicaciones, Universidad del Cauca, PopayánColombia, jflorez@unicauca.edu.co 


\section{Resumen}

Este artículo presenta el diseño, construcción y caracterización de un sistema automatizado de clasificación de producto en transportador de banda con visión de máquina que integra tecnología Free and Open Source Software y equipos comerciales Allen Bradley. Se definen requerimientos que determinan características: mecánicas de la estación de manufactura, de una aplicación clasificadora de productos con visión de máquina y de automatización del sistema. Para la aplicación de visión de máquina se usa la librería de procesamiento óptico digital de imágenes Open CV, para el diseño mecánico de la estación de manufactura se usa la herramienta CAD Solid Edge y para el diseño e implementación de la automatización se usan normas ISA junto a una metodología de proyectos de ingeniería en automatización integrando un PLC, un inversor, un Panel View y una Red DeviceNet. Se presentan pruebas de funcionamiento clasificando botellas y piezas de PVC en cuatro tipos establecidos, se comprueba el funcionamiento del sistema integrado y la eficiencia de la misma. El tiempo de procesamiento en visión de máquina es 0,290 s en promedio para una pieza de PVC, una capacidad de 206 accesorios $\mathrm{x}$ minuto, para botellas se logró un tiempo de procesamiento de $0,267 \mathrm{~s}$, una capacidad de 224 botellas x minuto. Se obtiene un rendimiento mecánico máximo de 32 productos por minuto (1920 productos hora) con el transportador a $22 \mathrm{~cm} / \mathrm{s}$ y con $40 \mathrm{~cm}$ de distancia entre productos obteniendo un error promedio de $0,8 \%$.

\section{Palabras clave}

Clasificación, visión de máquina, normas ISA, OpenCV, productos.

\section{Abstract}

This article presents the design, construction and testing of an automatic product sorting system in belt conveyor with machine vision that integrates Free and Open Source Software technology and Allen Bradley commercial equipment. Requirements are defined to determine features such as: mechanics of manufacturing station, an app of product sorting with machine vision and for automation system. For the app of machine vision a library is used for optical digital image processing Open $C V$, for the mechanical design of the manufacturing station is used the CAD tool Solid Edge and for the design and implementation of automation ISA standards are used along with an automation engineering project methodology integrating a PLC, an inverter, a Panel View and a DeviceNet Network. Performance tests are shown by classifying bottles and PVC pieces in four established types, the behavior of the integrated system is checked so as the efficiency of the same. The processing time on machine vision is $0.290 \mathrm{~s}$ on average for a piece of PVC, a capacity of 206 accessories per minute, for bottles was obtained a processing time of $0.267 \mathrm{~s}$, a capacity of 224 bottles per minute. A maximum mechanical performance is obtained with 32 products per minute (1920 products/hour) with the conveyor to $22 \mathrm{~cm} / \mathrm{s}$ and $40 \mathrm{~cm}$ of distance between products obtaining an average error of $0.8 \%$.

\section{Keywords}

Classification, machine vision, ISA norm, Open CV, products. 


\section{INTRODUCCIÓN}

En la automatización se intenta dotar a las máquinas de capacidades visuales, para que perciban la información de posición de objetos, el entorno, el movimiento, personas, midan características, calculen trayectorias, etc. [1]. Las tareas de percepción pueden ser ejecutadas con mayor objetividad y constancia por dispositivos electrónicos, que son más apropiados para realizar trabajos visuales altamente repetitivos $\mathrm{y}$ difíciles de efectuar por un operario [2]. Como en [3]-[6] hoy en día la visión de máquina es una disciplina en progreso con multitud de aplicaciones.

Por ejemplo en [7] realizan un sistema automatizado para clasificación de fresa a partir de: forma, tamaño y color, el sistema usa: cinta transportadora, cámara, sensores fotoeléctricos, entre otros componentes y es controlado por un chip-microcomputer (SMC) y PC. En [8] se desarrolla un sistema automatizado para la detección de papas irregulares, formado por cámara Sony, tarjeta Matrox Meteor, transportador de rodillos y PC. En [9]-[11] se realizan sistemas similares. En todos los trabajos anteriores se usan equipos electromecánicos y sistemas de visión comerciales de alto costo.

En Colombia, se han realizado trabajos en sistemas de automatización con visión de máquina. En [12] la PUJ desarrolló una metodología basada en visión para inspeccionar tolerancias geométricas y dimensionales de piezas mecanizadas en líneas de mecanizado. La Uninorte implementó un sistema Robot Vision Pro en un centro de manufactura para inspección de empaques de compresores centrífugos [13]. En la actualidad, se han difundido este tipo de aplicaciones en una amplia variedad de industrias a nivel nacional, donde empresas locales realizan soluciones con tecnología propia, por ejemplo Dakora [14] y Be Automation [15]. Sin embargo, contar a nivel académico con una estación de manufactura que permita diseñar y probar sis- temas de clasificación con visión de máquina es bastante complejo y costoso, se requiere una infraestructura física, capacitar laboratoristas y una alta inversión, por solo mencionar la estación de manufactura, difícilmente asequible en toda institución universitaria, otro tanto se requiere para adquirir un sistema de visión de máquina. Situación que debe ser sustentada en un plan académico y una serie de prácticas y laboratorios que permitan aprovechar al máximo la inversión.

El objetivo del presente proyecto es proponer una metodología de proyecto de ingeniería en automatización aplicada al diseño, construcción y caracterización de una estación de clasificación automática con visión de máquina, que integra tecnología FOSS (Free and Open Source Software) y equipos comerciales Allen Bradley, realizada en la Universidad del Cauca, que permite experimentar con sistemas de clasificación, inicialmente en uno de cuatro tipos establecidos para dos clases de productos.

En la sección 2 se desarrolla la metodología capturando requerimientos, diseñando la aplicación de visión de máquina, sistema mecánico e implementando la estación de clasificación según el modelado ISA S88, la sección 3 presenta los resultados de la caracterización del sistema y finalmente en la sección 4 se consignan las conclusiones.

\section{METOdOLOGía}

Se usa una metodología de proyectos de ingeniería en automatización desarrollada en el programa de Automática Industrial de la Universidad del Cauca. En su concepción se definen requerimientos que determinan características: mecánicas de la estación de manufactura, de una aplicación clasificadora de productos con visión de máquina y de automatización de todo el sistema. En el diseño e implementación de la automatización se usan normas ISA 
(S88 parte 1 y S5 partes 1 y 4) [18]. En la instrumentación se usa un PLC, un inversor, una pantalla táctil Panel View y una red DeviceNet todos Allen Bradley e integrados en línea a un sistema FOSS de visión de máquina. Finalmente se caracteriza la estación de clasificación a partir de la comparación del desempeño entre los sistemas de visión y mecánico

La estación de clasificación automática es un sistema integrado por un clasificador de productos de visión de máquina y una estación de manufactura. Para la aplicación de visión de máquina se usa la librería de procesamiento óptico digital de imágenes OpenCV [16], para el diseño mecánico de la estación de manufactura se usa la herramienta CAD Solid Edge v17 [17].

\subsection{Requerimientos de la estación de clasificación automática}

Realizar medición de características a dos clases de productos en movimiento sobre un transportador de banda: botellas plásticas con tapas de cuatro colores (azul, roja, blanca y verde) y accesorios de PVC de cuatro formas (cruz, te, codo y circular), para clasificarlos en cuatro tipos. A partir de condicionantes en la automatización fijados por el presupuesto asignado, equipos y tecnología disponible en los laboratorios, se organizan los requerimientos en tres grupos: visión de máquina, diseño mecánico y automatización del sistema.

En cuanto a visión de máquina los requerimientos son: usar un computador personal como módulo de procesamiento óptico digital de imágenes con FOSS; usar una cámara web de altas prestaciones como elemento de adquisición de imágenes; usar lenguaje $\mathrm{C}++$ y la librería OpenCV para diseñar e implementar scripts de procesamiento; diseñar scripts de procesamiento óptico digital de imágenes para cada producto; clasificar dos clases de productos: botellas plásticas y accesorios de PVC; clasificar cada clase de producto entre cuatro tipos establecidos: 1, 2, 3 y 4 (los tipos establecidos para las botellas son: tapas de cuatro colores: azul, roja, blanca y verde; los tipos establecidos para los accesorios de tubería de PVC son: cruz, te, codo y circular); y sincronizar la captura de la imagen por medio de un sensor que detecte el objeto sobre la banda.

En cuanto al diseño mecánico los requerimientos son: diseñar con dimensiones físicas moderadas para lograr su ubicación en un laboratorio convencional; reutilizar el transportador de banda del laboratorio de control de procesos; utilizar un esquema modular en el diseño de la planta de clasificación de piezas sobre el transportador de banda que permita una fácil construcción, instalación y mantenimiento; generar un flujo continuo y cerrado de piezas en la planta de clasificación; acumular mecánicamente las piezas en procesamiento con el fin de interrumpir el flujo y finalizar el proceso de clasificación; posicionar mecánicamente cada pieza en una de cuatro posibles posiciones a lo ancho de la banda de acuerdo a la decisión de clasificación; aplicar la normatividad de seguridad industrial necesaria; y contemplar la ubicación de paneles de cableado y un panel HMI.

En cuanto a la automatización del sistema los requerimientos son: indicar al usuario el script en ejecución correspondiente a la clase de producto procesado por medio de indicadores luminosos en la estación de manufactura e igualmente usando una pantalla Panel View V600Touch; usar como módulo de control en la estación de manufactura un PLC Allen Bradley Micrologix 1500; permitir la comunicación del módulo de control a la red de bus de campo DeviceNet de los laboratorios; enviar desde el PC hasta el PLC el tipo de producto resultante de la clasificación; ordenar por medio del PLC a un actuador mecánico la posición del producto en cuatro rangos de distancias según el tipo; operar a velocidad variable (entre 17 y $24 \mathrm{~cm} / \mathrm{s}$ ) usando un inversor; y usar normas técnicas industriales (ISA 88.1 e ISA s5.1/.4) para documentar el proyecto. 


\subsection{Clasificador de productos de visión de máquina}

Del análisis de los requerimientos, de visión de máquina y otros relacionados, se diseña e implementa una aplicación de visión de máquina para clasificar objetos de dos clases en uno de cuatro tipos prestablecidos. Esta obedece a etapas software y hardware (ver Fig. 1) que integran las componentes de procesamiento y análisis de imágenes necesarias para cumplir los requerimientos [18]. Inicia con la adecuación de la escena del producto en la banda, un sensor para iniciar la adquisición de la imagen por una cámara, un preprocesamiento y segmentación para extraer características del producto, reconocer y decidir sobre el tipo del mismo y comunicar al PLC quien ordena una acción física. En el anterior proceso intervienen elementos tanto hardware como software.

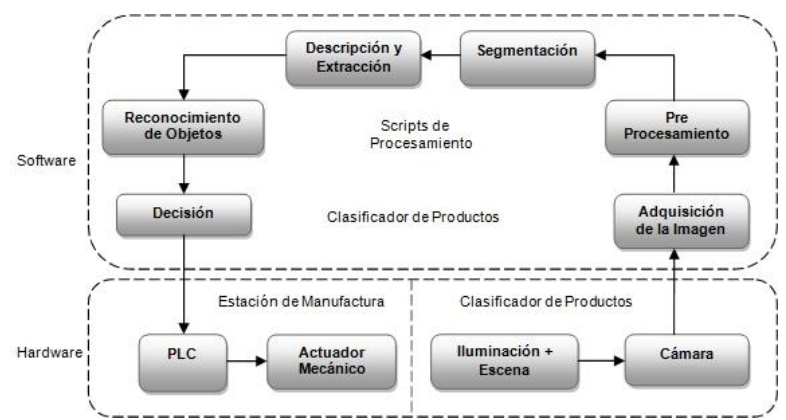

Fig. 1. Etapas software del clasificador de productos y hardware relacionado. Fuente: Autores

Los elementos software son el soporte lógico de la aplicación de visión de máquina, están compuestos principalmente por scripts de procesamiento (diseñados en lenguaje $\mathrm{C}++$ y OpenCV), junto a elementos necesarios para su ejecución: sistema operativo (Linux, Ubuntu 10.04, con las herramientas para desarrollo de aplicaciones), compilador (GCC "GNU Compiler Collection") y librerías (OpenCV "Open source Computer Vision library"), todos ellos presentes en un módulo de procesamiento.

Los elementos hardware desarrollan tareas de iluminación (Se utiliza la ilumina- ción propia del laboratorio, luz fluorescente en el techo), sensado de producto (trigger: sensor óptico infrarrojo que detecta la presencia de un producto frente a la cámara, y da inicio a la ejecución de la rutina de procesamiento de la imagen capturada), captura de imagen (cámara web Logitech C210 conectada por un puerto USB del módulo de procesamiento), ejecución de scripts (computador Qbex con puerto USB para la cámara y puerto paralelo para un circuito de interfaz con el trigger y el PLC) e interfaz con estación de manufactura (circuito electrónico que comunica el módulo de procesamiento con el trigger, pilotos indicadores y el PLC, usando un puerto paralelo.

Se realizan seis (6) etapas de procesamiento para cada tipo de producto: botellas plásticas y piezas de PVC [19]. Adquisición de imagen: implementada en $\mathrm{C}++$ con base en OpenCV, para diseñar e implementar scripts de procesamiento, que adecuan la imagen del producto obtenida por la cámara. Pre-procesamiento: para mejorar las imágenes adquiridas y obtener características para reconocer cada producto, ver Fig. 2. Segmentación: para las piezas de PVC se segmentó el canal rojo del espectro RGB, para las botellas plásticas se hizo segmentación de los tres canales, ver Fig. 3. Descripción y extracción: para cada producto se hizo diferente extracción de características; para las botellas, clasificadas por el color de la tapa, se usa el área de la imagen de cada canal RGB, con el promedio del área (pixeles) de cada tipo de botella en cada canal calculado con 20 imágenes de prueba; para las piezas de PVC clasificadas según la forma, se usa el promedio de las características área y perímetro de los cuatro tipos de piezas a partir de 20 imágenes de prueba. Reconocimiento de objetos: se realizó comparando el área obtenida de cada canal con un valor de referencia; este se determinó luego de obtener el promedio de área de cada canal, para cada tipo de producto, y la etapa final. Decisión, reconoce el color de la tapa, en el caso de 
las botellas o la forma en las piezas de PVC, para determinar el tipo de producto.

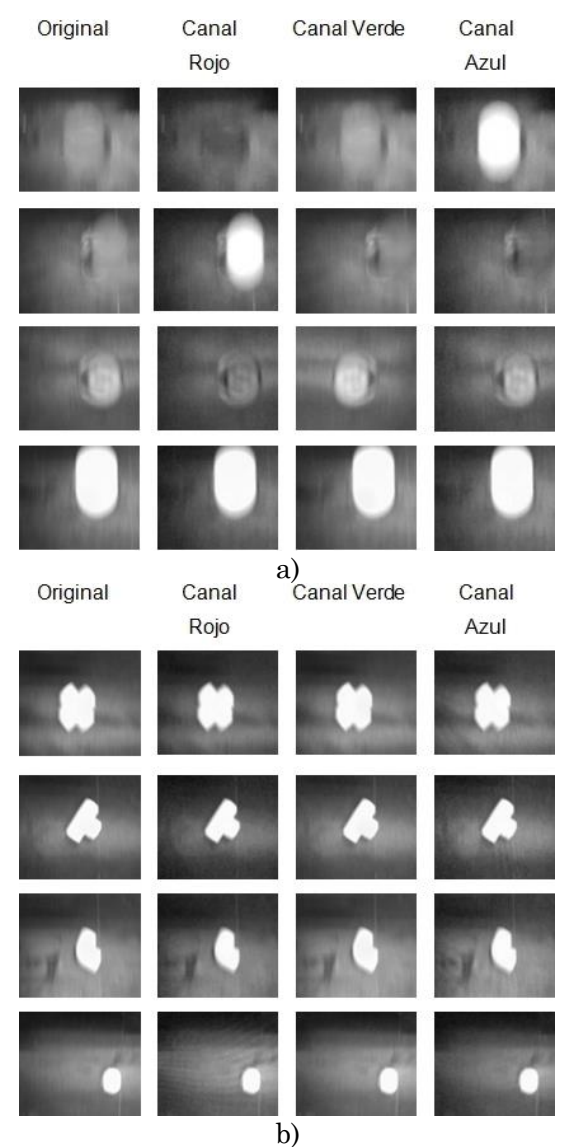

Fig. 2. Pre-procesamiento: a) para cada tipo botella y b) para cada pieza de PVC. Fuente: Autores

\subsection{Diseño y construcción mecánica}

Utiliza como base un transportador de banda ya existente en laboratorios, ver Fig. 4, compuesto de una estructura mecánica, una banda y un sistema motor AC; diseñada para soportar dos rodillos entre los cuales se tensa una banda de lona, el sistema motor gira uno de los rodillos, mueve la banda y por ende los objetos sobre esta.

Para cumplir los requerimientos de diseño mecánico se definen tres funciones principales a ejecutar de forma paralela en la estación de manufactura: Transporte de productos (traslada los productos por las etapas del proceso de clasificación), distribución de productos (ubica los productos en el centro del transportador de banda) y clasificación de productos (clasifica y tras- lada productos desde un extremo del transportador de banda hacia el otro formando un flujo continuo). A partir de los requerimientos de diseño mecánico y las funciones anteriores, se diseñan tres módulos mecánicos sobre el transportador de banda, usando la herramienta CAD Solid Edge, uno por cada función.

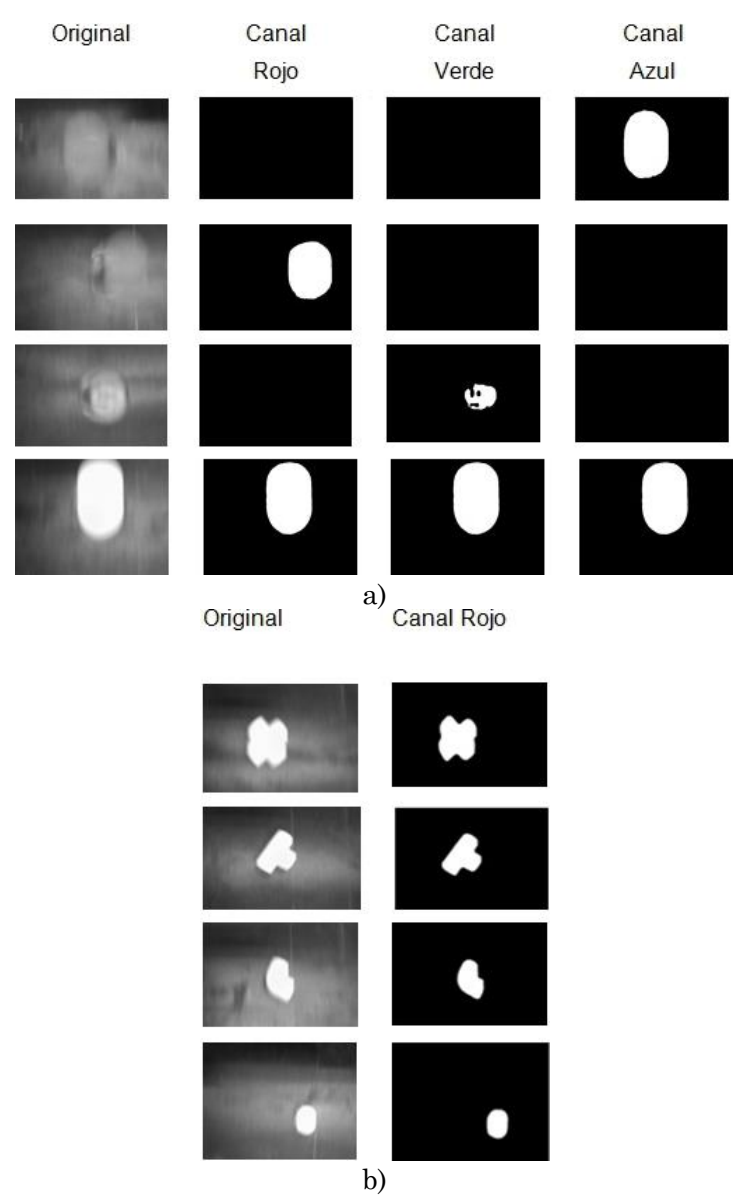

Fig. 3. Segmentación: a) para cada tipo botella y b) para cada pieza de PVC. Fuente: Autores

Módulo de Transporte: Traslada productos por las etapas de clasificación. Se compone de transportador de banda accionado por un motor trifásico, que se le diseño e instalo una banda y canal auxiliar. La banda auxiliar se ubica de forma paralela a la banda principal y genera un flujo continuo de productos; el canal auxiliar, estructura metálica que brinda soporte a la banda auxiliar, permite que los productos en movimiento se transporten sobre una superficie firme sin caerse, ver Fig. 5a. 


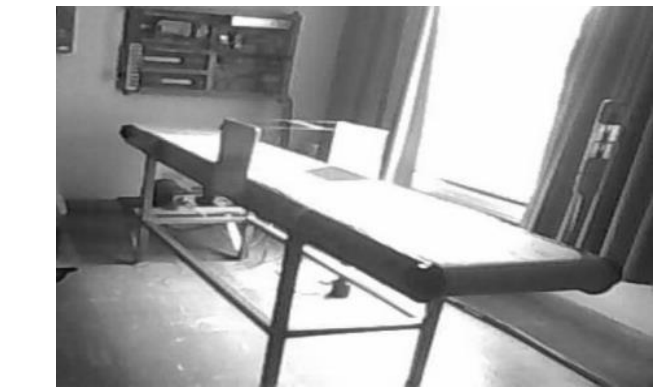

a)

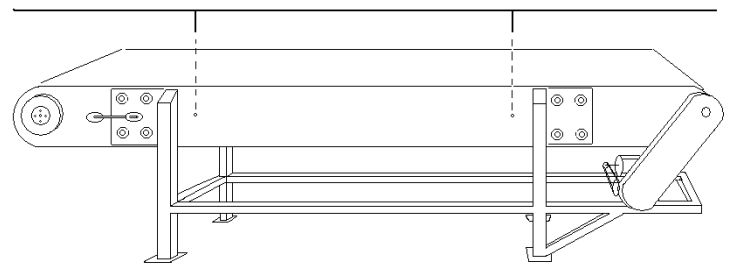

b)

Fig. 4. Transportador de banda, a) banda original, b) primer plano. Fuente: Autores

Módulo distribuidor: Traslada y ubica productos desde la banda auxiliar al centro de la banda principal. Compuesto por acumulador y distribuidor. El acumulador ubicado sobre la banda auxiliar retiene o no productos, está diseñado como una compuerta accionada por cilindro neumático. El distribuidor es un mecanismo compuesto por un conjunto de aspas que traslada productos desde la banda auxiliar hacia la parte central de la banda principal accionada por un motor AC, ver Fig. 5b.

Módulo de clasificación: Se compone de un clasificador y un recolector. El clasificador ubica los productos en una de cuatro posibles posiciones sobre la banda usando un cabezal accionado por cilindro neumático, ver Fig. 6. El recolector transporta los productos clasificados de la banda principal a la banda auxiliar, presenta el mismo diseño del distribuidor.

\subsection{Modelado ISA 88}

La norma ISA 88 provee una terminología estándar y modelos para plantas de manufactura y control batch [20]. Aunque no hay método definido para usar ISA88 en procesos no batch, los modelos y conceptos son aplicables, con variaciones de reglas y algunos requisitos, sin cambiar el modelo básico. Incluso se ha definido la extensión NS88 para aplicar los modelos ISA S88 en procesos continuos y discretos.

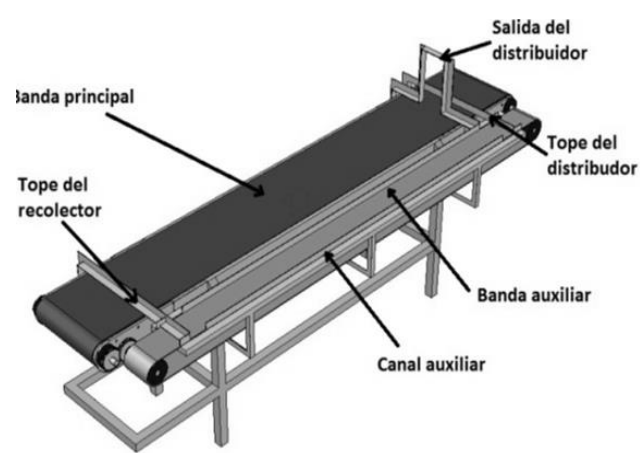

a)

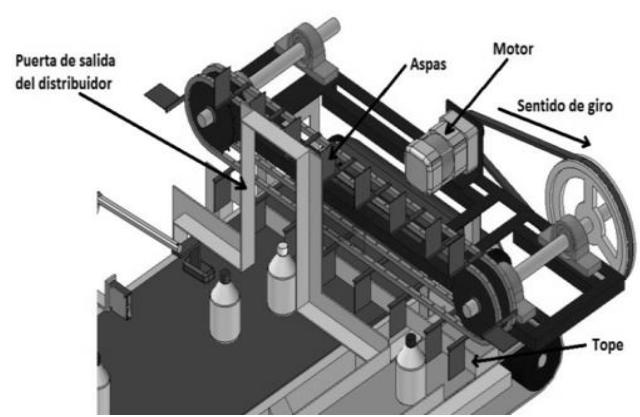

b)

Fig. 5. Vista isómetrica de los módulos: a) Trasporte, b) Distribuidor. Fuente: Autores
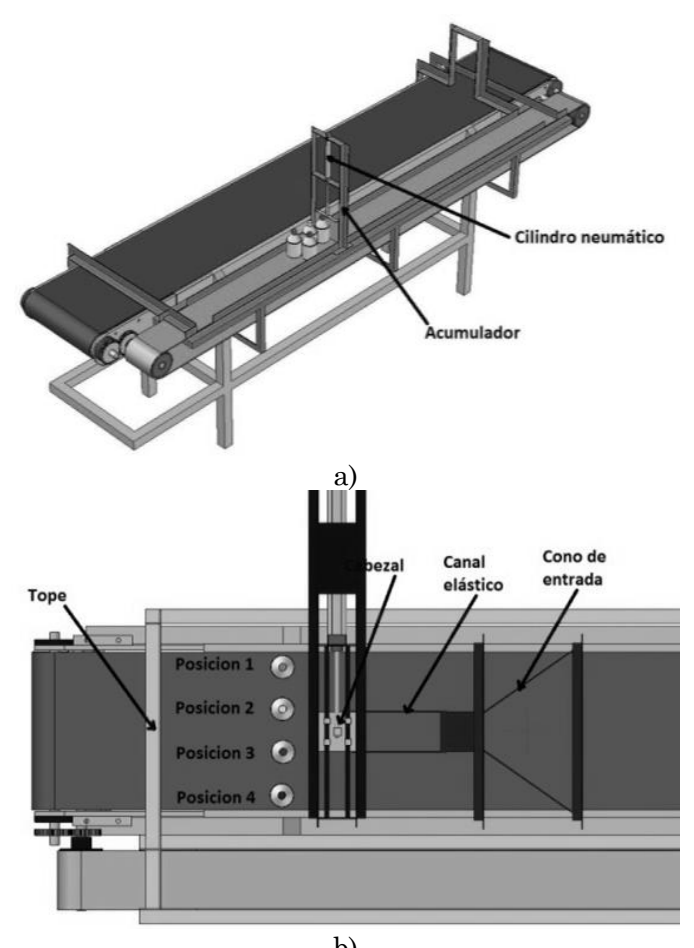

b)

Fig. 6. a) Posiciones de clasificación, b) Acumulador. Fuente: Autores 
En este trabajo se modela el proceso de clasificación de productos realizando tres modelos ISA 88: de proceso (ver Tabla 1), físico (ver Tabla 2) y de control procedimental (ver Tabla 3), donde la información se recopila y organiza, generando una base para la implementación de la Estación de Control de Calidad Automática, correspondiente a la instrumentación, control y supervisión de la misma [18].

\subsection{Instrumentación, control y supervisión}

Esquemas de control: De acuerdo a los modelos ISA 88 se identifican tres variables controladas: velocidad de la banda del transportador, paso de productos y posición de cada producto según su tipo. Para gobernarlas se usan como variables manipuladas: frecuencia y voltaje del motor trifásico, posición del acumulador y posición del cabezal del clasificador. Se identifican tres lazos de control: velocidad tipo feedback, cantidad de piezas tipo feedback y posición del clasificador tipo feedforward. Para cumplir con los requerimientos de automatización, se propone un escenario de automatización basado en PLC con red de bus de campo y monitoreo y supervisión local.

Instrumentación de la unidad de clasificación: El control del sistema de clasificación se realiza por medio de un PLC Micrologix 1500 de Allen Bradley y un bus de campo DeviceNet. El PLC es programado con software de Rockwell Automation, en él se ejecutan los algoritmos de control de los tres lazos del proceso de clasificación de productos.

Para monitorear y supervisar los lazos de control y el encendido - apagado del distribuidor, recolector, transportador y un compresor, se cuenta con dos HMI; el principal en una Panel View V600Touch y el otro en un panel HMI (HMI Box), que mediante indicadores luminosos y pulsadores permite un control local para encender y apagar: el distribuidor, el recolector, el transportador y un compresor.

Tabla 1. Modelo de proceso del proceso de clasificación de productos. Fuente: Autores

\begin{tabular}{llll}
\hline Proceso & Etapa & Operación & Acción \\
\hline & Transportar & $\begin{array}{l}\text { Determinar velocidad, Ajustar velocidad, Transportar productos } \\
\text { Determinar cantidad de productos a clasificar, Permitir paso de los } \\
\text { productos, Trasladar productos a banda principal }\end{array}$ \\
$\begin{array}{l}\text { Clasificación } \\
\text { de productos }\end{array}$ & Clasificación & Distribuir & $\begin{array}{l}\text { Determinar grupo de producto a clasificar, Detectar presencia de } \\
\text { producto frente a la webcam, Determinar tipo de producto }\end{array}$ \\
& & Posicionar pieza & $\begin{array}{l}\text { Detectar presencia de producto en clasificador, Determinar posición, } \\
\text { Cambiar trayectoria del producto, Trasladar producto a banda auxiliar }\end{array}$ \\
\hline
\end{tabular}

Tabla 2. Modelo físico del proceso de clasificación de productos. Fuente: Autores

\begin{tabular}{llll}
\hline $\begin{array}{l}\text { Celda de } \\
\text { proceso }\end{array}$ & Unidad & Módulo de equipo & Módulo de control \\
\hline $\begin{array}{l}\text { Sistema de } \\
\text { clasificación de } \\
\text { productos }\end{array}$ & $\begin{array}{l}\text { Unidad de } \\
\text { clasificación }\end{array}$ & Módulo de equipo de transporte de equipo de distribución & $\begin{array}{l}\text { Módulo de control de velocidad } \\
\text { Módulo de control de paso de productos } \\
\text { Motor AC monofásico 1 }\end{array}$ \\
& Módulo de equipo de posicionamiento & $\begin{array}{l}\text { Módulo de control de posición de productos } \\
\text { Motor AC monofásico 2 }\end{array}$ \\
\hline
\end{tabular}

Tabla 3. Modelo de control de procedimiento del proceso clasificación de productos. Fuente: Autores

\begin{tabular}{llll}
\hline Procedimiento & $\begin{array}{l}\text { Procedimiento de } \\
\text { unidad }\end{array}$ & Operación & Fase \\
\hline \multirow{2}{*}{$\begin{array}{l}\text { Clasificación de } \\
\text { productos }\end{array}$} & Clasificar & Adecuar transporte de productos & $\begin{array}{l}\text { Determinar velocidad, Ajustar velocidad } \\
\text { Determinar cantidad de productos, Permitir } \\
\text { paso de productos, Trasladar a banda principal }\end{array}$ \\
& Posicionar & $\begin{array}{l}\text { Determinar tipo de producto, Determinar posi- } \\
\text { ción, Cambiar trayectoria de producto, Trasladar } \\
\text { a banda auxiliar }\end{array}$ \\
\hline
\end{tabular}


A continuación se describe la instrumentación asociada a los lazos. Lazo de Control de Velocidad: para que el PLC haga monitoreo y control de la velocidad del motor se hizo uso de dos módulos, un contador de alta velocidad 1769 HSC para conectar un encoder y un módulo de señales analógicas 1769-IB4XOF2 para comunicar el esfuerzo de control al inversor, ver Tabla 4. El monitoreo y control de encendido y apagado del motor trifásico del transportador, se puede hacer tanto desde el panel HMI con dos pulsadores como desde el PLC, por medio de dos relés electromecánicos que van a entradas en el variador.

Tabla 4. Instrumentos lazos de control del proceso de clasificación. Fuente: Autores

\begin{tabular}{|c|c|c|}
\hline \\
\hline $\begin{array}{l}\text { Lazo de } \\
\text { control } \\
\end{array}$ & Función & Descripción \\
\hline \multirow{4}{*}{ Velocidad } & Controlador & PLC Micrologix 1500 \\
\hline & Pre actuador & Inversor trifásico Powerflex 40 \\
\hline & Actuador & Motor trifásico Baldor \\
\hline & Sensor & Encoder incremental DRC C152 \\
\hline \multirow{4}{*}{$\begin{array}{l}\text { Paso de } \\
\text { piezas }\end{array}$} & Controlador & PLC Micrologix 1500 \\
\hline & Pre actuador & Electroválvula neumática 5/2 \\
\hline & Actuador & $\begin{array}{l}\text { Cilindro neumático de doble } \\
\text { efecto de diámetro } 16 \mathrm{~mm} \mathrm{x} \\
200 \mathrm{~mm} \text { de recorrido }\end{array}$ \\
\hline & Sensor & $\begin{array}{l}\text { Sensor óptico en el clasificador } \\
\text { ( } 24 \text { V salida en colector abierto) }\end{array}$ \\
\hline \multirow{5}{*}{$\begin{array}{l}\text { Posición } \\
\text { de cada } \\
\text { pieza }\end{array}$} & Controlador & PLC Micrologix 1500 \\
\hline & Pre actuador & $\begin{array}{l}\text { Electroválvula neumática } 5 / 3 \text {, } \\
\text { centro cerrado y electroválvula } \\
\text { de apoyo }\end{array}$ \\
\hline & Actuador & $\begin{array}{l}\text { Cilindro neumático de doble } \\
\text { efecto de diámetro } 32 \mathrm{~mm} \mathrm{x} \\
350 \mathrm{~mm} \text { de recorrido }\end{array}$ \\
\hline & Sensor a & $\begin{array}{l}\text { Seis sensores ópticos para } \\
\text { ubicar el cabezal ( } 24 \mathrm{~V} \text { salida en } \\
\text { colector abierto) }\end{array}$ \\
\hline & Sensor b & $\begin{array}{l}\text { Sensor óptico en el clasificador } \\
\text { ( } 24 \mathrm{~V} \text { salida en colector abierto) }\end{array}$ \\
\hline
\end{tabular}

Lazo de control de paso de piezas: el sensor óptico ubicado a la entrada del clasificador permite contar la cantidad de piezas que van pasando por el proceso, de forma que cuando se ha completado la cantidad total, por medio del cilindro neumático y la electroválvula neumática se cambia la posición del acumulador entre abierto y cerrado. El PLC realiza el monitoreo y control de la cantidad de piezas en el proceso, ver Tabla 4.

Lazo de control de posición de cada pieza: el PLC le indica a la aplicación de visión el grupo de piezas que se procesará; la aplicación de visión determina el tipo de pieza y envía la decisión al PLC cuando el sensor b se activa, ver Tabla 4. El PLC lee la señal del tipo de pieza, al igual que la señal de seis sensores ópticos que indican la posición actual del cabezal; de esta forma se determina la dirección en que debe moverse el cabezal manipulando la posición del pistón del cilindro, por medio de una electroválvula neumática, de forma que las piezas sean clasificadas correctamente. El PLC realiza el monitoreo y control de la posición de cada pieza según el tipo.

Comunicación de PLC con bus de campo y diagrama de proceso e instrumentos P\&ID: la comunicación del PLC con el Panel View V600 y con el adaptador DeviceNet 1734-ADN se realiza empleando el protocolo e interfaz DeviceNet a una velocidad de transferencia de $125 \mathrm{kbits} / \mathrm{s}$, por medio del scanner 1769-SDN conectado al PLC Micrologix 1500. En la Fig. 7 se presenta el diagrama P\&ID de los lazos de control del proceso de clasificación y en la Fig. 8 los HMI del sistema.

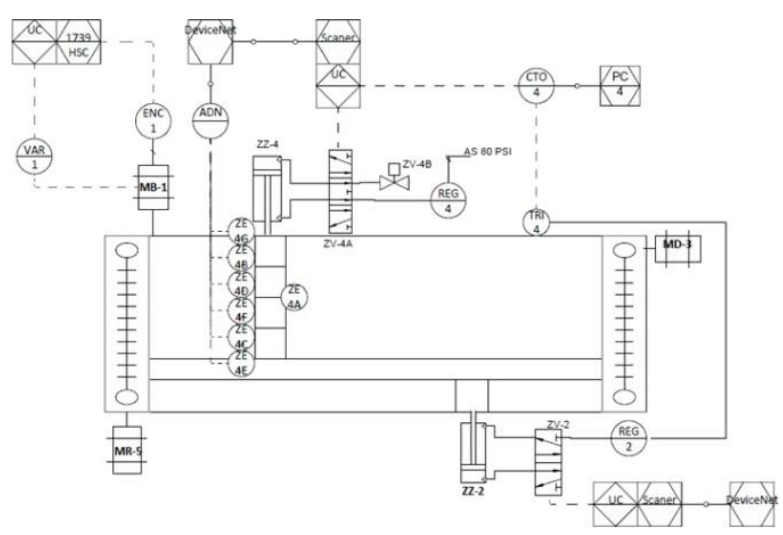

Fig. 7. Diagrama P\&ID del sistema de clasificación de piezas. Fuente: Autores 

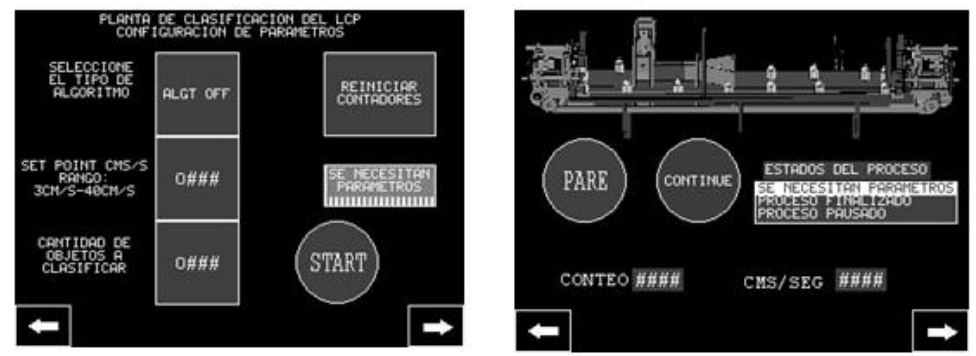

(a)

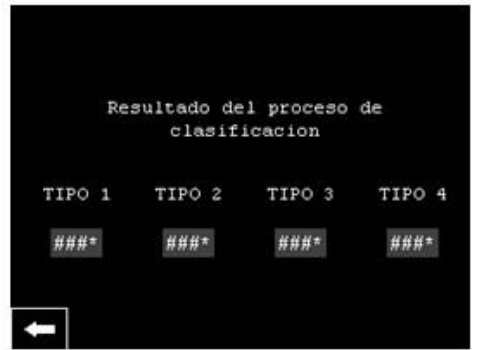

4

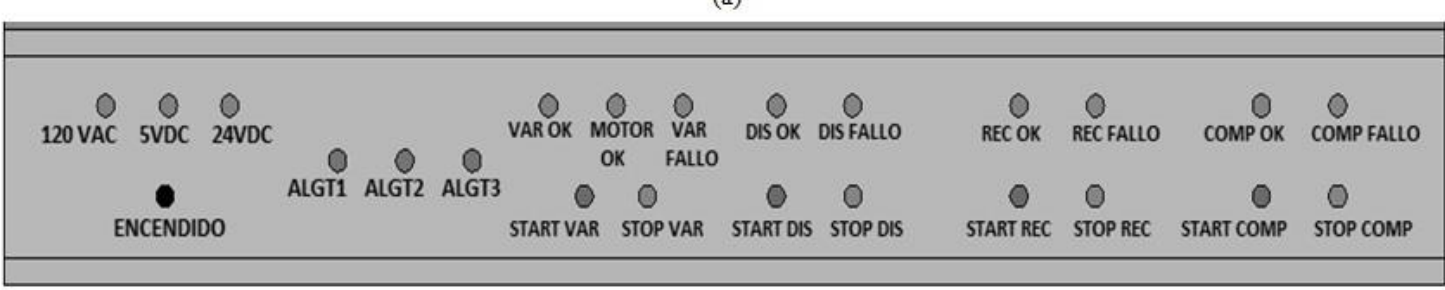

(b)

Fig. 8. HMI: a) Pantallas en Panel View V600, b) Interfaz en tablero de control. Fuente: Autores

\section{RESULTADOS Y DISCUSIÓN}

Se realizan pruebas individuales para medir el rendimiento de los scripts de procesamiento. En cuanto a las botellas, se evalúa el desempeño de los scripts de clasificación capturando imágenes de botellas con la banda en movimiento a $22 \mathrm{~cm} / \mathrm{s}$, y verificando su tipo. Se capturaron 40 imágenes en tiempo real, diez de cada tipo, con banda en movimiento, se usaron los scripts correspondientes para clasificar cada una de las imágenes, y se comparó si el resultado obtenido correspondía al tipo de pieza real. En la Tabla 5 se consignan los datos luego de clasificar 40 imágenes de botellas, con banda en movimiento. En las filas se encuentra el número real de botellas de cada color, y en las columnas el resultado de clasificación, el reconocimiento de productos dio resultado correcto con la banda a $22 \mathrm{~cm} / \mathrm{s}$ con porcentaje error de $2,5 \%$.

En cuanto a los accesorios de PVC, se evalúa el desempeño de los scripts de clasificación capturando imágenes de accesorios de PVC con la banda en movimiento a 18 $\mathrm{cm} / \mathrm{s}$, y verificando su tipo. Se capturaron 52 imágenes en tiempo real, 13 de cada tipo, con banda en movimiento, se usaron los scripts correspondientes para la clasificación, y se comparó si el resultado obtenido correspondía al tipo de pieza real. En la Tabla 6 se consignan los datos de clasificar 52 imágenes, con banda en movimiento. En las filas se encuentra el número real de tipos de accesorios de PVC, y en las columnas el resultado de clasificación, el reconocimiento de productos dio porcentaje de error de $0 \%$ con la banda a $18 \mathrm{~cm} / \mathrm{s}$.

Tabla 5. Ficha y resultados de prueba de clasificación botellas. Fuente: Autores

\begin{tabular}{lccccc}
\hline & Roja & Azul & Verde & Blanca & $\begin{array}{c}\text { Cantidad } \\
\text { real }\end{array}$ \\
\hline Tapa Roja & 10 & 0 & 0 & 0 & 10 \\
Tapa Azul & 0 & 10 & 0 & 0 & 10 \\
Tapa Verde & 0 & 1 & 9 & 0 & 10 \\
Tapa Blanca & 0 & 0 & 0 & 10 & 10 \\
$\begin{array}{l}\text { Resultado de } \\
\text { Clasificación }\end{array}$ & 10 & 11 & 9 & 10 & Total: \\
\hline
\end{tabular}

Tabla 6. Ficha y resultados de prueba de clasificación de piezas de PVC. Fuente: Autores

\begin{tabular}{lccccc}
\hline & Cruz & Codo & Circular & Te & $\begin{array}{c}\text { Cantidad } \\
\text { Real }\end{array}$ \\
\hline Cruz & 13 & 0 & 0 & 0 & 13 \\
Codo & 0 & 13 & 0 & 0 & 13 \\
Circular & 0 & 0 & 13 & 0 & 13 \\
Te & 0 & 0 & 0 & 13 & 13 \\
Resultado de & 13 & 13 & 13 & 13 & $\begin{array}{l}\text { Total: } \\
\text { Clasificación }\end{array}$ \\
\hline
\end{tabular}


En las pruebas de clasificador de productos en estación de manufactura se midió la capacidad de procesamiento del clasificador de productos en la estación de manufactura, ver Fig. 9, como un sistema integrado de clasificación, determinando la cantidad de productos por minuto que clasifica, el porcentaje de error y las limitaciones generales.

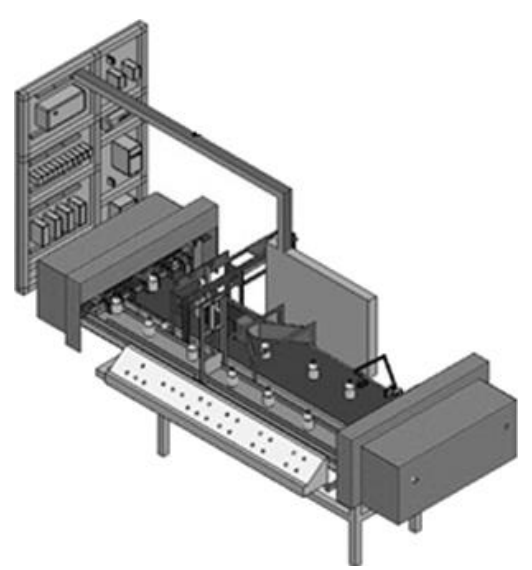

a)

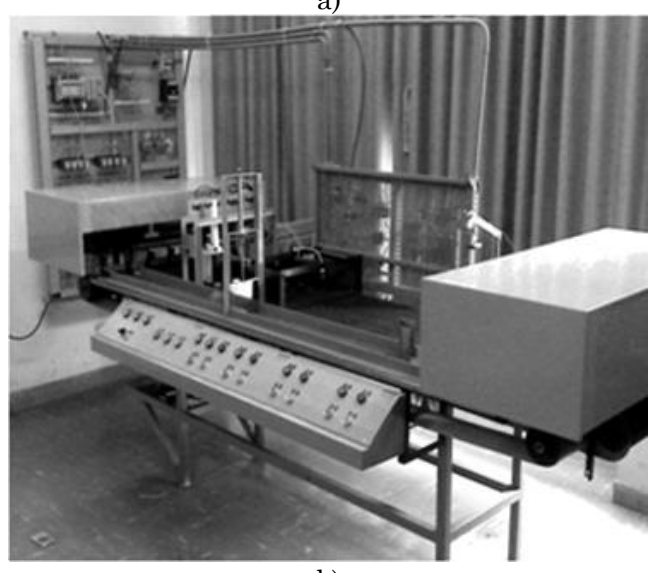

b)

Fig. 9. Sistema de clasificación de productos, a) Modelo $\mathrm{CAD}$, b) Montaje final. Fuente: Autores

\subsection{Prueba productos por minuto}

Se identificaron y midieron los tiempos críticos del sistema: Tiempo de captura de imagen (tiempo que tarda en procesarse la orden de capturar una nueva imagen), tiempo de procesamiento de imagen (tiempo que consumen los scripts en procesar cada imagen hasta generar una decisión de clasificación), tiempo de estabilización PLC (es el tiempo que demora en estabilizarse las entradas del PLC que reciben la decisión de clasificación desde el circuito de interfaz) y tiempo de desplazamiento mecánico (es el tiempo que tarda en moverse el actuador entre las cuatro posiciones). Se midieron los tiempos críticos asociados a la aplicación de visión y a la estación de manufactura, para determinar el rendimiento total del sistema clasificador. Se consideraron las siguientes condiciones: Distancia entre productos de $40 \mathrm{~cm}$ y velocidad de la banda entre 17 y $22 \mathrm{~cm} / \mathrm{s}$. La Tabla 7 consigna los tiempos críticos medidos.

Tabla 7. Resultados clasificador de productos en la estación de manufactura. Fuente: Autores

\begin{tabular}{|c|c|c|c|c|}
\hline \multicolumn{3}{|c|}{ Clasificador de productos } & \multicolumn{2}{|c|}{ Estación de manufactura } \\
\hline $\begin{array}{l}\text { Captura } \\
\text { imagen }\end{array}$ & $\begin{array}{l}\text { Procesa } \\
\text { imagen }\end{array}$ & $\begin{array}{l}\text { Espera } \\
\text { para leer } \\
\text { PLC }\end{array}$ & $\begin{array}{c}\text { Espera leer } \\
\text { entrada } \\
\text { PLC }\end{array}$ & $\begin{array}{l}\text { Mueve } \\
\text { actuador }\end{array}$ \\
\hline $0,010 \mathrm{~s}$ & $\begin{array}{c}0,207 \mathrm{~s} \\
\text { (Bot) } \\
0,230 \mathrm{~s} \\
\text { (PVC) }\end{array}$ & $0,050 \mathrm{~s}$ & $1 \mathrm{~s}$ & $\begin{array}{l}0,39 \mathrm{~s} \\
\text { (Min.) } \\
0,84 \mathrm{~s} \\
\text { (Máx.) }\end{array}$ \\
\hline \multicolumn{3}{|c|}{206 productos $\mathrm{x}$ minuto } & \multicolumn{2}{|c|}{32 productos $\mathrm{x}$ minuto } \\
\hline \multicolumn{5}{|c|}{$\begin{array}{c}32 \text { productos } \mathrm{x} \text { minuto }(40 \mathrm{~cm} \text { de distancia entre productos, } \\
\text { Vel. banda }=22 \mathrm{~cm} / \mathrm{s} \text { ) }\end{array}$} \\
\hline
\end{tabular}

Para el clasificador de productos, en el peor de los casos el tiempo que tarda en clasificar un producto es de $0,290 \mathrm{~s}(0,01+$ $0,23+0,05)$, permitiendo un límite teórico de 206 accesorios de PVC x minuto. Mientras que para clasificar una botella el clasificador de productos tarda 0,267 s $(0,01+$ $0,207+0,05)$, permitiendo un límite teórico de 224 botellas x minuto (14 mil productos hora). Para la estación de manufactura el peor de los casos tarda $1,840 \mathrm{~s}(1,0+0,84)$, teniendo en cuenta la espera de $1 \mathrm{~s}$ programada para evitar leer decisiones incorrectas, y dar tiempo para que los productos lleguen al actuador permitiendo un límite teórico de 32 productos x por minuto, y limitando la velocidad de procesamiento del sistema.

\subsection{Pruebas de ejecución continúa}

Se realizaron 5 pruebas de clasificación de productos con el sistema integrado en 
ejecución continua, contando los productos en los que falla la clasificación, y determinando si el error proviene del clasificador de productos o de la estación de manufactura. Las condiciones para la prueba son: Distancia entre productos mínimo $40 \mathrm{~cm}$ y velocidad de banda entre 17 y $22 \mathrm{~cm} / \mathrm{s}$. La Tabla 8 consigna el porcentaje error de cada prueba, se obtiene un error promedio global del 0,8\%, los errores presentados se deben a falla en el clasificador de productos y el segundo a falla en la estación de manufactura. La Tabla 9 consigna los parámetros de funcionamiento adecuado del clasificador de productos en la estación de manufactura y otras consideraciones.

\begin{tabular}{ccccc}
\multicolumn{5}{c}{$\begin{array}{c}\text { Tabla } \\
\text { 8. Pruebas finales del clasificador de productos en } \\
\text { estación de manufactura. Fuente: Autores }\end{array}$} \\
\begin{tabular}{ccclc} 
Prueba & Productos & Error & \multicolumn{1}{c}{ Motivos } & Error \\
\hline 1 & 50 & 1 & $\begin{array}{l}\text { Se clasifico mal un } \\
\text { producto }\end{array}$ & $2 \%$ \\
2 & 50 & 1 & $\begin{array}{l}\text { El clasificador no se } \\
\text { movió }\end{array}$ & $2 \%$ \\
3 & 50 & 0 & - & $0 \%$ \\
4 & 50 & 0 & - & $0 \%$ \\
5 & 50 & 0 & - & $0 \%$ \\
\hline
\end{tabular}
\end{tabular}

Tabla 9. Parámetros de clasificación y consideraciones del sistema integrado. Fuente: Autores

\begin{tabular}{ll}
\hline \multicolumn{2}{c}{ sistema integrado. Fuente: Autores } \\
\hline $\begin{array}{l}\text { Parámetro } \\
\text { Cantidad total de productos a }\end{array}$ & Ilimitada \\
$\begin{array}{l}\text { Cantidad máxima de } \\
\text { productos en el sistema }\end{array}$ & 8 \\
$\begin{array}{l}\text { Velocidad de la banda } \\
\text { Distancia mínima entre } \\
\text { productos }\end{array}$ & $18-24 \mathrm{~cm} / \mathrm{s}$ \\
\hline Consideración & $40 \mathrm{~cm}$ \\
\hline $\begin{array}{l}\text { Altura de la lente con respecto } \\
\text { a la banda }\end{array}$ & $30 \mathrm{~cm}$ \\
Inclinación trigger (botellas) & $\begin{array}{l}\text { Hacia arriba, configurar } \\
\text { flanco de subida }\end{array}$ \\
$\begin{array}{l}\text { Inclinación trigger (accesorios } \\
\text { PVC) }\end{array}$ & $\begin{array}{l}\text { Hacia abajo, configurar } \\
\text { flanco de bajada }\end{array}$ \\
\hline
\end{tabular}

\section{CONCLUSIONES}

Se diseñó e implementó un sistema clasificador de productos con visión de máquina en una estación de manufactura haciendo uso de una metodología que permitió integrar tecnología FOSS con equipos comerciales Allen Bradley, capaz de clasificar dos clases de productos entre cuatro tipos establecidos, botellas plásticas con tapas de cuatro colores: azul, roja, blanca y verde y piezas de PVC con cuatro formas: cruz, te, codo y circular. Se describieron las componentes de procesamiento y análisis de imagen: Adquisición imagen implementada en lenguaje $\mathrm{C}++$ con base en la librería OpenCV, para diseñar e implementar los scripts de procesamiento; preprocesamiento que permite el mejoramiento de las imágenes adquirida; segmentación, para los tipos de accesorios de PVC, se segmentó en el canal rojo del espectro RGB, en el caso de las botellas plásticas, se hizo la segmentación de los tres canales. Descripción y extracción, para cada grupo de producto se hizo una extracción de características diferentes; área para botellas y área perímetro para piezas de PVC. Reconocimiento de objetos, se realizó comparando el área obtenida de cada canal con un valor de referencia, y decisión, al reconocer el color de la tapa en el caso de las botellas, o la forma en el caso de los accesorios de PVC, se determinar el tipo de producto.

En las pruebas individuales para las botellas plásticas se capturaron 40 imágenes en tiempo real con la banda a $20 \mathrm{~cm} / \mathrm{s}$, clasificándolas y verificando su tipo: azul, roja, verde y blanca; el porcentaje de error obtenido fue de $2,5 \%$ con la banda a 21,7 $\mathrm{cm} / \mathrm{s}$. Para los accesorios de PVC se capturaron 52 imágenes en tiempo real con la banda a $21,7 \mathrm{~cm} / \mathrm{s}$, clasificándolos y verificando su tipo: cruz, codo, circular y te; se clasificaron todas las imágenes con la banda a $18 \mathrm{~cm} / \mathrm{s}$ con porcentaje de error de $0 \%$.

En las pruebas del sistema integrado, el tiempo de procesamiento del clasificador de productos es $0,290 \mathrm{~s}$ en promedio para un accesorio de PVC, lo cual da una capacidad de 206 accesorios de PVC x minuto (12 mil productos hora). Para las botellas el tiempo promedio que tarde el clasificador de productos es $0,267 \mathrm{~s}$, lo cual da una capacidad de 224 botellas $\mathrm{x}$ minuto (14 mil productos hora). 
En general, la capacidad del sistema depende de la velocidad de la banda de la estación de manufactura y de la distancia entre los productos, obteniendo un rendimiento máximo de 32 productos por minuto (1920 productos hora) con la banda a 22 $\mathrm{cm} / \mathrm{s}$ y con $40 \mathrm{~cm}$ de distancia entre productos obteniendo un error promedio de $0,8 \%$.

\section{AGRADECIMIENTOS}

Se agradece al Departamento de Electrónica, Instrumentación y Control de la Universidad del Cauca por financiar el presente proyecto.

\section{REFERENCIAS}

[1] R. de la Rosa, "Procesamiento de Imágenes Digitales," in X Congreso Nacional en Informática y Computación del Instituto Tecnológico de Puebla, 2007.

[2] A. Escalera, Visión por computador (fundamentos y métodos). Pearson - Prentice Hall, 2001, p. 304.

[3] L. de J. Mesa-Palacio, G. Calle-Trujillo, and J. J. Arbeláez-Toro, "Diseño, Construcción y Fabricación de una Máquina Prototipo de Medición por Coordenadas," Tecno Lógicas, no. 26, pp. 11-25, 2011.

[4] D. E. Guyer, G. E. Miles, M. M. Schreiber, O. R. Mitchell, and V. C. Vanderbilt, "Machine Vision and Image Processing for Plant Identification," Trans. $A S A E$, vol. 29, no. 6, pp. 1500-1507, 1986.

[5] R. P. Wildes, J. C. Asmuth, G. L. Green, S. C. Hsu, R. J. Kolczynski, J. R. Matey, and S. E. McBride, "A machine-vision system for iris recognition," Mach. Vis. Appl., vol. 9, no. 1, pp. 1-8, Jan. 1996.

[6] S. Kovačič and F. Lahajinar, "A Machine Vision System for the Rotational Positioning and Verification of Oil Filters," Int. J. Adv. Manuf. Technol., vol. 21, no. 6, pp. 452-459, Mar. 2003.

[7] X. Liming and Z. Yanchao, "Automated strawberry grading system based on image processing," Comput. Electron. Agric., vol. 71, pp. S32-S39, Apr. 2010.

[8] G. ElMasry, S. Cubero, E. Moltó, and J. Blasco, "Inline sorting of irregular potatoes by using automated computer-based machine vision system," J. Food Eng., vol. 112, no. 1-2, pp. 60-68, Sep. 2012.

[9] J. Blasco, S. Cubero, J. Gómez-Sanchís, P. Mira, and E. Moltó, "Development of a machine for the automatic sorting of pomegranate (Punica granatum) arils based on computer vision," J. Food Eng., vol. 90, no. 1, pp. 27-34, Jan. 2009.

[10] W. Zhang, J. Mei, and Y. Ding, "Design and Development of a High Speed Sorting System Based on Machine Vision Guiding," Phys. Procedia, vol. 25, pp. 1955-1965, Jan. 2012.

[11] H. Raafat and S. Taboun, "An integrated robotic and machine vision system for surface flaw detection and classification," Comput. Ind. Eng., vol. 30, no. 1, pp. 27-40, Jan. 1996.

[12] G. M. Zambrano, C. A. Parra, M. R. Manrique, and C. J. Bustacara, "Estación de control de calidad por visión artificial para un centro de manufactura integrada por computador (CIM)," Ing. y Univ., vol. 11, no. 1, pp. 33$55,2007$.

[13] R. López, E. Sotter, and E. Zurek, "Aplicación del sistema Robot Vision PRO para operaciones automáticas de control de calidad," Rev. Científica Ing. y Desarro., vol. 9, no. 9, pp. 88-97, 2001.

[14] Dakora, "Dakora Automatización Industrial," 2013. [Online]. Available: http://www.dakora.com.co/. [Accessed: 01-May-2013].

[15] B. Automation, "Be Automation," 2013. [Online]. Available: http://www.beautomation.com.co/. [Accessed: 01-May-2013].

[16] Intel® Software Network, "Open Source Computer Vision Library (OpenCV)." 2013.

[17] Siemens PLM Software, "Solid Edge." 2013.

[18] M. Pérez and O. Velasco, "Sistema de clasificación de piezas en una línea de producción empleando visión de máquina," Universidad del Cauca, 2011.

[19] R. C. González and P. Wintz, Procesamiento digital de imágenes. Addison-Wesley, 1996.

[20] American National Standard Institute, "ANSI/ISAS88.01-1995," 2006. 